\title{
F. Dostoevskij, Lettere, a cura di A. Farina, trad. di G. De Florio, A. Farina, E. Freda Piredda, Il Saggiatore, Milano 2020, pp. 1376.
}

Il volume Lettere del Saggiatore, curato da Alice Farina, raccoglie 438 lettere indirizzate da Fëdor Michajlovič Dostoevskij a un eterogeneo novero di destinatari (prevalentemente, familiari, amici, editori, redattori, scrittori, critici letterari). Pur mancando le lettere/repliche (dei destinatari allo scrittore), si tratta di una parte fondamentale dell'epistolario dostoevskiano, quasi esaustiva rispetto al corpus accessibile in lingua russa. L'interesse dell'opera è dato anche dal fatto che numerose lettere vengono qui pubblicate per la prima volta in lingua italiana. La traduzione dal russo è firmata dalla curatrice Farina (Responsabile Ufficio Stampa e Comunicazione del Saggiatore), da Giulia De Florio (Ricercatrice, russista) e Elena Freda Piredda (Dottoranda, russista). La traduzione è accompagnata da un sobrio apparato di note a piè di pagina. Le lettere sono numerate in progressione cronologica sia nel testo, sia nel Sommario iniziale, che funge da indice dei contenuti e che occupa 19 pagine (non numerate). Seguono l'introduzione della Curatrice e, subito dopo, gli Appunti di traduzione (firmati da due delle tre traduttrici: De Florio e Piredda; pp. 39-49). L'opera si chiude con l'Indice dei nomi, utile per accedere alle lettere in base ai destinatari o ai riferimenti al loro interno (pp. 1359-1370), e con l'Indice delle opere di Dostoevskij (menzionate, si sottintende, nei testi epistolari, pp. I37I-1372).

Pubblicate nell'autunno del 2020, le Lettere di Fëdor Dostoevskij costituiscono uno dei più corposi contributi editoriali italiani connessi al bicentenario della nascita dello scrittore russo (nato a Mosca il 30-X/II-XI I82I), ricorrenza che ha avuto risonanza nel mondo intero. L'ambizioso progetto, si deduce dall'insieme, mirava ad attirare l'attenzione dei lettori italiani interessati all'opera e/o alla figura di Dostoevskij, ma privi di accesso linguistico alle fonti russe. In tal senso, al di là dell'opportuna coincidenza con il bicentenario, il progetto si è attenuto ad alcuni fondamentali parametri dei long seller: personaggio famoso, materiali inediti, casa editrice prestigiosa, veste editoriale 'solida'. Manca solo il rigore, cioè il parametro che avrebbe garantito all'opera non solo la 'visibilită, ma anche la qualità duratura che rende un libro un riferimento obbligato per chiunque, esperto e non, cerchi un lavoro rigoroso. Infatti, è difficile che slavisti o studiosi di mestiere possano affidarsi senza riserve a questa edizione, in quanto non sono stati rispettati gli standard accademico-scientifici: l'affidabilità epistemologica, storico-critica e traduttologica. Chi ha curato il progetto editoriale ha scelto di non avvalersi della consulenza di studiosi esperti di Dostoevskij, del genere epistolare, della traduzione. Dispiace poiché si trattava di un'eccezionale opportunità per creare un'opera di alto profilo, in grado di fornire quello "strumento di studio" cui Farina esplicitamente allude nell'introduzione (p. 23). Il volume, invece, suscita notevoli perplessità a livello progettuale e procedurale. 
Al lettore più esigente l'apparato scientifico appare limitato nella forma e dilettantesco nella sostanza. L'introduzione, dal titolo ad effetto (Il romanzo di una vita, pp. 23-38), è priva di elaborazione concettuale ed estranea alle consuetudini accademiche (coerenza e ordine espositivo). Senza argomentare la sue idee, Farina equipara l'epistolario a uno "strumento per interpretare Dostoevskij e la sua opera" (p. 26, corsivo mio), convinta (senza alcuna consapevolezza critica) che "le lettere fungono per Dostoevskij [...] da vero punto di partenza creativo, da fonte inesauribile di verità" ( $\mathrm{p}$. 27 , corsivo mio). Si tratta di sentenze ingenue (e, per uno studioso serio, anche irritanti) che palesano l'estraneità di Farina ai numerosi studi sul genere letterario epistolare: nell'arco di mezzo secolo è stata ampiamente argomentata a livello scientifico l'incompatibilità tra epistolari e "verità" (concetto, peraltro, estraneo alla terminologia scientifica). Non c'è alcun cenno nell'Introduzione non solo ai singoli saggi e articoli sull'argomento, ma neppure alle fondamentali monografie di studiosi di chiara fama (come N. Bonifazi, S. Hubier, V. Kaufmann, P. Lejeune, J. Starobinski). Sebbene alcune idee meno ingenue, con debiti approfondimenti, avrebbero incuriosito, sebbene si succedano citazioni e riferimenti (che vanno, senza logica consequenzialità, da Hitchcock a Berdjaev, da Hugo a Girard), le esigue pagine introduttive di Farina ammiccano in modo pretestuale (e anche in questo caso, parrebbe, inconsapevole) a un desueto soggettivismo post-moderno. Si rilevano frasi prive di coerenza contestuale e di senso compiuto (ad es., "La storia di una vita è sempre stata la storia di un corpo. Il corpo che lo si voglia o no, è vessillo e sospiro, compagno esigente, mappa del nostro presente e del nostro futuro"; p. 27). Per quanto riguarda il corpus, la tipologia e le fonti delle lettere, la Curatrice si limita a poche, vaghe parole sul criterio di selezione: "si è scelto di pubblicare qui la quasi totalità delle lettere. Sono state escluse soltanto le missive che nulla avrebbero aggiunto" (p. 26). Nulla si dice sul Testo di Partenza russo in nessuna parte del volume.

Non meno deludenti e confuse appaiono le pagine degli Appunti di traduzione, poiché predispongono il lettore ad aspettarsi le fondamentali informazioni sul progetto traduttivo, sulle strategie, sulle principali difficoltà incontrate, mentre si tratta di una 'seconda introduzione' che, pur migliore della prima, crea false aspettative. Neppure qui, comunque, si riflette sulla specifica complessità del genere epistolare. Parlando della scrittura dostoevskiana, infatti, le autrici trascurano le cruciali differenze tra generi testuali che, nel caso di Dostoevskij, sono al centro d'importanti dibattiti; inoltre, contraddicono Farina stessa quando attribuiscono una "frequente intenzione manipolatoria" alla "nervosa" scrittura dostoevskiana (pp. 40-4I): per quanto non venga definita (e sia concettualmente subdola), l'idea di un' "intenzione manipolatoria" contraddice quella di Farina, cioè che le lettere di Dostoevskij siano un deposito di "verità".

Quanto alla traduzione dal russo, la sistematica resa della sintassi e della fraseologia non marcate del testo russo con quelle marcate dell'italiano (ad es., "per l'amor di Dio" invece di "mi raccomando", "ci nominano" invece di "ci chiamano", "in nome di Dio" invece di "per carità, "Che Dio sia con loro!", invece di "Buon per loro!” [cioè, "vadano a farsi benedire!]”, "la sua opinione degli uomini" invece di "la sua opinione sugli esseri umani", "siamo stati amareggiati dal sapere" invece di "ci ha amareggiato venire a sapere") rende faticosa e sgradevole la lettura di queste lettere, così come a) i ripetuti calchi dei pronomi non marcati in russo, resi con ridondanti e obsoleti pronomi marcati in italiano (disturbano, soprattutto, esso/essa/esse/essi) e b) i calchi della punteggiatura russa, la cui asimmetria rispetto a quella italiana è fatto noto e vistoso anche al di fuori della traduttologia. Le traduttrici, lettera dopo lettera, impongono al lettore italiano il sintagma vistosamente marcato dall'esclamativo “Caro/a __ !" che calca il russo non marcato “Dorogoj/aja___?, il quale corrisponde (senza eccezioni) all'italiano non marcato “Caro/a__,. In questa formula epistolare, l'equivalenza esclamativo $[R u] \Leftrightarrow$ virgola [It] non è materia di opinione o di gusto. Per quanto riguarda le varie 
forme ipocoristiche derivate da antroponimi russi presenti nelle lettere - quasi sempre opache e ostiche agli stranieri - le traduttrici le calcano senza nulla fare, né dire al lettore, come se il problema (ben studiato sia in onomastica, sia in traduttologia) non esistesse. La responsabilità di questa traduzione - che avrebbe richiesto ancora un lungo e capillare lavoro di revisione - spetta senza dubbio alla Curatrice, sia perché chi 'cura' risponde sempre del progetto editoriale nel suo complesso, sia perché Farina ha direttamente partecipato alla traduzione.

Per quanto, infine, riguarda la veste materiale delle Lettere, si riscontrano pregi e difetti. Nonostante l'ottima leggibilità dei caratteri, l'apprezzabile veste tipografica e la buona qualità della carta, il libro è troppo voluminoso e pesante; ha, infatti, le dimensioni di un corposo dizionario monovolume, come se funzione dell'opera fosse di essere 'consultata' e non 'letta', il che andrebbe bene se la Curatrice non avesse sostenuto l'ipotesi che queste lettere ("romanzo di una vita") vadano lette come un'"opera letteraria”. Anche la copertina rigida, che pur favorisce l'impatto estetico e la conservazione del libro, rende difficile gestire la lettura senza un appoggio. La versione in due volumi avrebbe avvantaggiato la lettura.

In sintesi, se l'editore avesse mirato alla qualità del progetto e del libro, cioè al suo rigore e al conseguente prestigio scientifico, avrebbe inevitabilmente fatto ricorso a consulenti di mestiere: letterati, affermati studiosi di Dostoevskij, esperti di traduzione. Il volume, invece, appare impostato e realizzato in modalità prettamente amatoriale, ma ben al di sotto dello standard (più elevato) dei pionieri della slavistica di metà $\mathrm{xx}$ secolo (che erano almeno eruditi interpreti). È davvero inspiegabile che oggi, nel 2021, nel momento in cui la qualità delle opere tradotte sta raggiungendo i più alti standard professionali sul mercato editoriale italiano, si sia optato per un approccio così 'approssimativo'. Anche a voler ragionare in base a criteri meramente commerciali, come sa qualsiasi 'imprenditore', deludere i 'clienti esigenti' non è mai una scelta saggia. 